\title{
HOMOTOPIC ARCS ARE ISOTOPIC
}

\author{
C. D. FEUSTEL ${ }^{1}$
}

In the following all spaces are simplicial complexes and all maps are piecewise linear. A simple arc $\alpha$ is regularly embedded in a compact 2-manifold $M$ if the intersection of $\alpha$ with the boundary of $M$ contains exactly the endpoints of $\alpha$. Two simple arcs $\alpha$ and $\beta$ with common endpoints $p$ and $q$ are isotopic with endpoints fixed if there exists a continuous family of homeomorphisms $h_{t}, 0 \leqq t \leqq 1$, mapping $M$ onto $M$ such that $h_{0}$ is the identity, $h_{1}(\beta)=\alpha, h_{t}(p)=p$, and $h_{t}(q)=q$.

It is the purpose of this note to prove:

TheOREM. Let $\alpha$ and $\beta$ be simple arcs regularly embedded in a compact 2-manifold $M$ and having common endpoints $p$ and $q$. Let $\alpha$ and $\beta$ intersect in only a finite number of points. If $\alpha$ and $\beta$ are homotopic, they are isotopic.

The author wishes to thank E. M. Brown for posing this problem and for many helpful conversations of both a mathematical and a stylistical nature.

Let us briefly consider the restrictions on $\alpha$ and $\beta$. It is not necessary that $\alpha$ and $\beta$ be polygonal arcs nor that they intersect only a finite number of times. The theorem is, however, false if the arcs are not required to be regularly embedded. Figure 1 illustrates that homotopic arcs with common endpoints may fail to be isotopic although each lies wholly in the interior of the manifold.

Throughout the remainder of this note $\alpha, \beta, p, q$, and $M$ will be as in the theorem. $T$ will be tubular neighborhood of $\alpha$; that is $T$ is a subset of $M$ homeomorphic to $(-1,1) \times \alpha$ under a homeomorphism $\phi$ such that $\phi(0 \times \alpha)=\alpha$ and $\phi(1-1,1) \times\{p, q\})$ are contained in the boundary of $M$. The components of $T-\alpha$ will be denoted $T_{1}$ and $T_{2}$. A subloop of $\alpha$ and $\beta$, (subloop), is a simple closed curve formed by a segment of $\alpha$ and a segment of $\beta$. We shall only consider subloops which are determined by intersection points consecutive with respect to a parameterization of $\beta$. An intersection point $x$ of $\alpha$ and $\beta$ will be called a crossing point of $\alpha$ and $\beta$ if either $x$ is an endpoint of $\alpha$ and $\beta$ or $\alpha$ crosses from one side of $\beta$ to the other at $x$. Note that if $\alpha$ crosses $\beta$ at $x$, then $\beta$ also crosses $\alpha$ at $x$.

Received by the editors November 11, 1965.

1 This work was supported by the Office of Naval Research Grant Nonr. 3897(02). The author would like to thank the referee for his comments. 


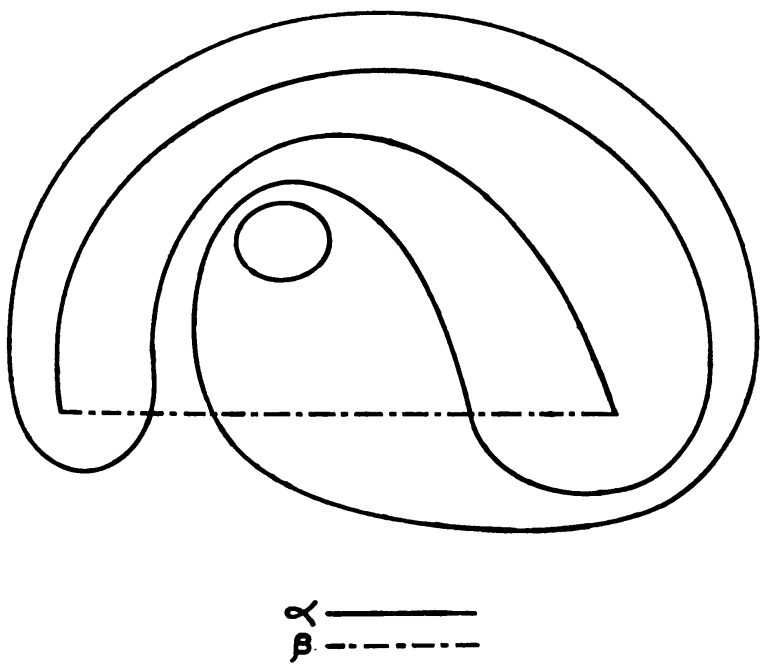

Figure 1. Arcs in an annulus.

The theorem will be proved by showing:

(1) Intersection points which are not crossing points can be removed isotopically without introducing new intersection points. In fact, if $x$ is an intersection point of $\alpha$ and $\beta$ which is not a crossing point, it is possible to construct an isotopy which is the identity on the complement of a neighborhood $N_{x}$ of $x$ pulling $\beta$ away from $\alpha$ at $x$. Choosing $N_{x}$ sufficiently small prevents the isotopy from introducing new intersection points.

(2) When $\alpha$ and $\beta$ intersect in more than two points, each intersection being a crossing point, an isotopy can be found transforming at least one crossing point into an intersection point, not a crossing point. This isotopy will not introduce new intersection points nor will it move $p$ or $q$.

(3) When $\alpha$ and $\beta$ intersect exactly in two crossing points, i.e., their endpoints, an isotopy exists which satisfies the conditions of the theorem.

Isotopies of forms (1) and (2) are found inductively until only two intersection points remain. The isotopy is finished using (3). Thus the proof of the theorem has been reduced to proving statements (1), (2), and (3).

The proof of statements (2) and (3) falls naturally in to the same two sections. In the first a special set of generators for $\pi_{1}(M, p)$ will be chosen. These generators will be used to show that $\alpha$ and $\beta$ have a trivial subloop, that is, a subloop homotopic to a point. In the 
second section, it will be shown that a trivial subloop bounds a disk and that the isotopy required in either statement can be accomplished by deforming $\beta$ across this disk.

1. Construction of the generators. It will be convenient to adopt a standard parameterization of $\alpha$ and $\beta$ at this point. Let $\alpha(t), \beta \vec{r}(t)$ be fixed parameterizations of $\alpha$ and $\beta$ such that $\alpha \vec{c}(0)=\beta^{\rightarrow}(0)=p$ and $\alpha^{\rightarrow}(1)=\beta^{\rightarrow}(1)=q$. Let $\alpha^{\leftarrow}(t)=\alpha^{\rightarrow}(1-t)$ and $\beta^{\leftarrow}(t)=\beta^{\rightarrow}(1-t)$. Let the symbol $\beta^{\rightarrow} \alpha^{-}$be the closed path obtained by following $\beta^{\rightarrow}$ with $\alpha^{\leftarrow}$. Let $\lambda_{i}$ be the subloop of $\alpha$ and $\beta$ determined by $\beta^{\rightarrow}\left(t_{i}\right)$ and $\beta^{\rightarrow}\left(t_{i+1}\right)$; these being the $i$ th and $i+1$ th intersection points along $\beta$. Let $l_{i}$ be the loop obtained by tracing $\alpha$ from $p$ to $\beta \rightarrow\left(t_{i}\right)$, tracing $\beta$ from $\beta \rightarrow\left(t_{i}\right)$ to $\beta \rightarrow\left(t_{i+1}\right)$, and finally tracing $\alpha$ from $\beta\left(t_{i+1}\right)$ to $p$. Note that $l_{i}$ is freely homotopic to $\lambda_{i}$. We shall denote by $\left[l_{i}\right]$ the element of $\pi_{1}(M, p)$ represented by $l_{i}$. We remark that $\prod_{i}\left[l_{i}\right]=\left[\beta^{\rightarrow} \alpha^{-}\right]$. Now we shall say that $l_{i}$ ends in $T_{j}$ for $j=1,2$, if for $t$ less than but sufficiently near $t_{i+1}, \beta \rightarrow(t) \in T_{j}$. Likewise $l_{i}$ begins in $T_{j}$ if for $t$ greater than but sufficiently near $t, \beta \rightarrow(t) \in T_{j}$.

Let us assume that $M$ is separated by $\alpha$. Let $x_{1}$ and $x_{2}$ be the closures of the components of $M-\alpha$. Let $\left\{V_{1} \cdots V_{n}\right\}$ be a system of loops which lie in $x_{1}$ such that $\left\{\left[V_{1},\right], \cdots,\left[V_{j}\right]\right\}$ is a system of free generators for $\pi_{1}\left(x_{1}, p\right)$ and let $\left\{V_{j+1}, \cdots, V_{n}\right\}$ be the same in $x_{2}$. Since $x_{i}$ for $i=1,2$, is a 2 -manifold with boundary, $\pi_{1}\left(x_{i}, p\right)$ is a free group. Applying van Kampen's theorem, we see that $\left\{\left[V_{1}\right] \ldots\right.$ $\left.\left[V_{n}\right]\right\}$ is a free system of generators for $\pi_{1}(M, p)$ since $\pi_{1}\left(x_{1} \cap x_{2}, p\right)$ is a trivial group. In case $\alpha$ separates $M$, this will be our free system of generators for $\pi_{1}(M, p)$.

Let us assume that $M-\alpha$ is connected. Let $x_{1}$ be $M-T_{2}$. Let $\mu$ be a path which lies in $x_{1}$ and irreducibly joins the two components of the intersection of $x_{1}$ and the closure of $T_{2}$. Let $x_{2}$ denote the union of $\mu$ and the closure of $T_{2}$. By van Kampen's theorem the union of a free set of generators for $\pi_{1}\left(x_{1}, p\right)$ and a free set of generators for $\pi_{1}\left(x_{2}, p\right)$ is a free set of generators for $\pi_{1}(M, p)$. Note that $\pi_{1}\left(x_{2}, p\right)$ is a free group since $x_{2}$ has the homotopy type of a circle. Let $V_{1}$ be a simple loop originating at $p$, traversing $\alpha$ from $p$ to the intersection of $\alpha$ and $\mu$ next traversing $\mu$, and finally passing through $T_{2}$ to $p$. Then $\left[V_{1}\right]$ generates $\pi_{1}\left(x_{2}, p\right)$. Choose a set of simple loops $\left\{V_{2} \cdots V_{n}\right\}$ embedded in $x_{1}$ such that $\left\{\left[V_{2}\right] \cdots\left\{V_{n}\right\}\right\}$ is a free set of generators for $\pi_{1}\left(x_{1}, p\right)$. Now $\left\{\left[V_{1}\right], \cdots,\left[V_{n}\right]\right\}$ is a free set of generators for $\pi_{1}(M, p)$. This will be our free set of generators for $\pi_{1}(M, p)$ if $M-\alpha$ is connected.

Lemma 1. Let $M-\alpha$ be connected. Assume that $\lambda_{k}$ is a nontrivial sub- 
loop of $\alpha$ and $\beta$. The loop $l_{k}$ ends in $T_{2}$ if and only if the reduced word for $\left[l_{k}\right]$ ends with $\left[V_{1}\right]$. The loop $l_{k}$ begins in $T_{2}$ if and only if the reduced word $\left[l_{k}\right]$ begins with $\left[V_{1}\right]^{-1}$.

Proof. Assume that $l_{k}$ begins in $T_{1}$ and ends in $T_{2}$. The loop $\left[l_{k}\right]\left[V_{1}\right]^{-1}$ is homotopic to a loop in $x_{1}$. Thus $\left[l_{k}\right]\left[V_{1}\right]^{-1}$ is a word in the generators $\left[V_{2}\right], \cdots,\left[V_{n}\right]$, and the reduced word for $\left[l_{k}\right]$ ends in $\left[V_{1}\right]$.

The remaining three cases in the proof are similar.

LEMMA 2. Assume that each intersection point of $\alpha$ and $\beta$ is a crossing point. Then $\alpha$ and $\beta$ have a trivial subloop.

Proof. Assume to the contrary that no $\left[l_{i}\right]=1$. Since $\left[\beta^{\rightarrow} \alpha^{\leftarrow}\right]=1$, there exists a $k$ such that formal cancellation occurs between the words for $\left[l_{k}\right]$ and $\left[l_{k+1}\right]$. It follows from the definition of crossing point that if $l_{k}$ ends in $T_{2}, l_{k+1}$ begins in $T_{1}$ and conversely.

Case 1. Assume $M-\alpha$ is connected. Assume that $l_{k}$ ends in $T_{2}$. By Lemma $1,\left[l_{k}\right]$ ends in $\left[V_{1}\right]$ and $\left[l_{k+1}\right]$ does not begin in $\left[V_{1}\right]^{-1}$. This contradicts our assumption of formal cancellation. The proof is similar if $l_{k}$ ends in $T_{1}$.

Case 2. Assume $\alpha$ separates $M$. A subloop lies in $x_{1}$ or $x_{2}$. If $\lambda_{k}$ lies in $x_{1}$, then $l_{k+1}$ lies in $x_{2}$ and $\left[l_{k}\right]$ and $\left[l_{k+1}\right]$ are words in $\left[V_{1}\right] \cdots\left[V_{j}\right]$ and $\left[V_{j+1}\right] \cdots\left[V_{n}\right]$ respectively. But then there can be no formal

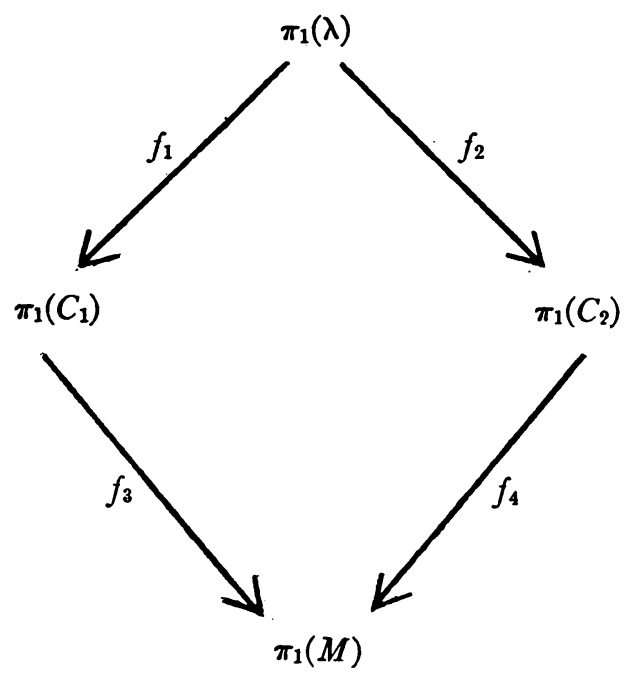

DIAGRAM 1 
cancellation between $\left[l_{k}\right]$ and $\left[l_{k+1}\right]$. We have a contradiction. The proof is similar if $l_{k}$ lies in $x_{2}$.

\section{Construction of the isotopy.}

LEMMA 3. Let $\lambda$ be a simple closed curve embedded in $M$ and homotopic to a point. Then $\lambda$ bounds a disk, proved in less generality in [2].

Proof. Assume that $\lambda$ is interior to $M$. Since $\lambda$ is homotopic to a point, it is homologous to zero. Thus there exists a submanifold $C_{1}$ of $M$ bounded by $\lambda$. Let $C_{2}$ be the closure of the complement of $C_{1}$. Assume that neither $C_{1}$ nor $C_{2}$ is a disk, then it is easy to see that $\lambda$ is not homotopic to a point in either $C_{1}$ or $C_{2}$. It follows that $f_{1}$ and $f_{2}$ (induced by inclusion) in Diagram 1 are one-one.

According to a lemma of Brown and Crowell [1], $f_{3}$ and $f_{4}$ (induced by inclusion) are also 1-1. But then $\lambda$ is not homotopic to a point in $\pi_{1}(M)$. It follows that $C_{1}$ or $C_{2}$ must have been a disk.

If $\lambda$ is not interior to $M$, construct a manifold $M_{1}$ by joining an annulus to each component of the boundary of $M$ whose intersection with $\lambda$ is not void. Then $\lambda$ is interior to $M_{1}$ and, by the above argument, $\lambda$ bounds a disk in $M_{1}$. This disk is contained in $M$ since it does not meet the boundary of $M_{1}$.

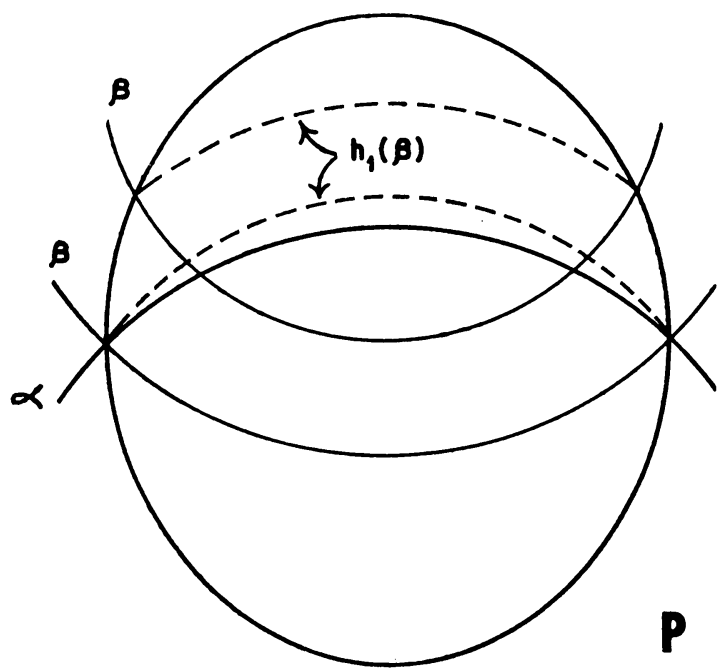

FIGURE 2. 
Assume that $\alpha$ and $\beta$ have more than two intersection points, all being crossing points. Let $\lambda_{k}$ be a trivial subloop of $\alpha$ and $\beta$. By Lemma $3, \lambda_{k}$ bounds a disk, $D$. We observe that all points of $\alpha$ contained in $D$ lie on its boundary. Let $P$ be disk which is a neighborhood of $D-\left\{\beta\left(t_{k}\right), \beta\left(t_{k+1}\right)\right\}$. Let $\beta\left(t_{k}\right)$ and $\beta\left(t_{k+1}\right)$ be on the boundary of $P$. Choose $P$ so small that the component of $P-D$ with $\alpha \cap \lambda_{k}$ on its boundary contains no points of $\alpha$. Further the component of $P-D$ with $\beta \cap \lambda_{k}$ on its boundary should not meet $\beta$. Choose an isotopy $h_{t}$ leaving points outside of $P$ fixed and moving $\beta \cap \lambda_{k}$ across $D$ and beyond $\alpha$. See Figure 2. Then $h_{1}(\beta(t))=\beta(t)$ or $h_{1}(\beta(t))$ lies in the component of $P-D$ containing no points of $\alpha$. Therefore we have introduced no new intersection points. Also either $\beta\left(t_{k}\right)$ or $\beta\left(t_{k+1}\right)$ is no longer a crossing point. Statement (2) follows.

Assume that $\alpha$ and $\beta$ intersect in exactly two points, $p$ and $q$. Let $P$ and $D$ be as above and choose an isotopy $h_{t}$ leaving points outside of $P$ fixed and moving $\beta$ across $D$ and onto $\alpha$. Statement (3) follows.

\section{BIBLIOGRAPHY}

1. E. M. Brown and R. H. Crowell, The augmentation subgroup of a link, J. Math. Mech. 15 (1966).

2. H. I. Levine, Homotopic curves on surfaces, Proc. Amer. Math. Soc. 14 (1963), 986.

Dartmouth College and INDIANA UNIVERSITY 